\title{
SOME COMMENTS ON NARROW BAND-PASS FILTERS*
}

\author{
BY \\ M. ROSENBLATT \\ Brown University
}

1. Introduction. It appears to be part of the engineering folklore that a narrow band-pass filter applied to a stationary random input yields an output that is approximately normally distributed. Of course, such a conjectured result could not be true in absolute generality. At the very least, one ought to require ergodicity of the random process being filtered. However, we should like to sketch out a domain within which such a result would in fact hold and indicate roughly boundaries outside of which such normality would not be expected.

2. Preliminary discussion. Consider $x(t),-\infty<t<\infty$, a strictly stationary separable (see Doob [1] p. 52) random process. The separability is assumed so that meaningful statements can be made about sample functions (or realizations) of the process. Later on, a mixing condition and various moment conditions will be introduced and assumed to hold for $x(t)$. Our interest will be centered about weighted averages

$$
\int_{0}^{T} w_{T}(t) x(t) d t
$$

of a certain form. Though we shall actually deal with a more general context, the weight function $w_{T}(t)$ can be thought of as the weighting introduced by the filter. The assumptions on $w_{T}(t)$ will be such that it is amenable to a generalized Fourier analysis (see [2] p. 233). Set

$$
W=W(T)=\int_{0}^{T} w_{T}^{2}(t) d t
$$

An explicit indication of the dependence of $W$ on $T$ will of ten be deleted in the rest of the paper

The conditions on $w_{T}(t)$ will be called assumptions $(A)$.

Assumptions ( $A)$.

1. $W(T) \rightarrow \infty$ as $T \rightarrow \infty$

2. The functions $w_{T}(t)$ are slowly increasing in that

a) $\quad \int_{A(T)}\left|w_{T}(t)\right|^{2} d t=o(W)$ as $T \rightarrow \infty$

for any sequence of subsets $A(T)$ of $[0, T]$ with the Lebesque measure of $A(t) m(A(T))=$ $o(T)$, uniformly in $m(A(T)) / T$, as $T \rightarrow \infty$ and

b) $\quad w_{T}(t)=0\left(W^{-1 / 2}\right)$ uniformly in $t$

*Received March 14, 1960. The work presented in this paper was carried out on behalf of the Bell Telephone Laboratories. The author gratefully acknowledges their support and encouragement. 
as $T \rightarrow \infty$

3.

$$
\lim _{T \rightarrow \infty} W^{-1} \int_{0}^{T-|h|} w_{T}(t+|h|) w_{T}(t) d t=\rho(h)
$$

exists for every $h$ and is continuous in $h$.

The limit function $\rho(h)$ can be seen to be a non-negative definite function and therefore by Bochner's theorem ([4] p. 207) it has a representation

$$
\rho(h)=\int_{-\infty}^{\infty} e^{i \hbar \lambda} d M(\lambda)
$$

with $M(\lambda)$ a non-decreasing bounded function. The spectral mass of $M(\lambda)$ is symmetrically located about zero since $\rho(h)=\rho(-h)$. When $w_{T}(t)$ corresponds to a bandpass filter, conditions 1 and 2 of $(A)$ will usually be obviously satisfied since the functions $w_{T}(t)$ are typically uniformly bounded in $T$ with $W(T)$ of the same order of magnitude as $T$. If one is narrow band-pass filtering about $\mu, M(\lambda)$ will increase only at $\mu$ and $-\mu$. The conditions on $w_{T}(t)$ have been taken somewhat more general than required in bandpass filtering so as to cover situations that commonly occur in discussions of regression problems as they arise in time series analysis ([2], p. 233).

The process $x(t)$ will be assumed to satisfy a strong mixing condition. Let $B_{t}$ be the Borel field of events generated by the random variables $x(u), u \leq t$, and $\mathcal{F}_{\tau}$ the Borel field of events generated by the random variables $x(u), u \geq \tau$. Thus $B_{t}$ and $\mathcal{F}_{\tau}$ represent the information given by knowledge of the process before $t$ and after $\tau$ respectively.

Assumption (B). The process $x(t)$ satisfies the strong mixing condition $(B)$ if there is some positive function $g(u)$ defined for $0 \leq u<\infty$ with $g(u) \downarrow 0$ as $u \rightarrow \infty$ such that for any pair of events $B \varepsilon B_{t}, F \varepsilon F_{\tau}, t<\tau$,

$$
\left|P\left(B \cap F^{\prime}\right)-P(B) P(F)\right|<g(\tau-t) .
$$

The mixing condition $(B)$ might be called a uniform mixing condition. It is appreciably stronger than ergodicity and somewhat stronger than what one ordinarily calls mixing ([3] p. 36). We shall later see by virtue of an example that ergodicity alone is not enough to guarantee that the output of a narrow band-pass filter be normal.

As in the case of any central limit theorem, certain moment conditions are necessary. The conditions to be specified are certainly not the best that could be obtained but they are quite natural in the context we are talking about. Assume that fourth moments $E x(t)^{4}$ exist and that the process $x(t)$ is continuous in the mean of fourth order, that is,

$$
E|x(t)-x(z)|^{4} \rightarrow 0
$$

as $t \rightarrow \tau$. It is convenient to take the constant first moment $E x(t)$ as identically zero. Because of the stationary of $x(t)$, the mixed fourth order moment

$$
E\left[x\left(t_{1}\right) x\left(t_{2}\right) x\left(t_{3}\right) x\left(t_{4}\right)\right]=P\left(t_{2}-t_{1}, t_{3}-t_{1}, t_{4}-t_{1}\right)
$$

depends only on the differences $t_{2}-t_{1}, t_{3}-t_{1}, t_{4}-t_{1}$. Let $R(t)$ denote the covariance

$$
R\left(t_{2}-t_{1}\right)=E\left[x\left(t_{1}\right) x\left(t_{2}\right)\right] \text {. }
$$

We introduce the fourth order cumulant function

$$
\begin{array}{r}
Q\left(t_{2}-t_{1}, t_{3}-t_{1}, t_{4}-t_{1}\right)=P\left(t_{2}-t_{1}, t_{3}-t_{1}, t_{4}-t_{1}\right) \\
-P_{G}\left(t_{2}-t_{1}, t_{3}-t_{1}, t_{4}-t_{1}\right),
\end{array}
$$


where $P_{G}$ is what $P$ would be in the case of a normal process, nainoly

$$
\begin{aligned}
P_{G}\left(t_{2}-t_{1}, t_{3}-t_{1}, t_{4}-t_{1}\right)=R\left(t_{2}-t_{1}\right) R\left(t_{4}-t_{3}\right)+R\left(t_{3}-t_{1}\right) R\left(t_{4}-t_{2}\right) \\
+R\left(t_{4}-t_{1}\right) R\left(t_{3}-t_{2}\right)
\end{aligned}
$$

(see Magness' paper [5]). Notice that if $R(t)$ is absolutely integrable, the spectral density $f(\lambda)$ of the process exists and is continuous.

Assumption $(C) . R(t)$ and $Q\left(t_{1}, t_{2}, t_{3}\right)$ are absolutely integrable over one and three dimensional space respectively. The spectral density $f(\lambda)$ is assumed to be positive everywhere. If one were only considering narrow band-pass filtering about $\mu$, the positivity of $f(\lambda)$ would only be required at $\mu$. It should also be noted that the proof of asymptotic normality to be given does not use the stationarity of $x(t)$ fully, only stationarity up to moments of fourth order.

3. The basic theorem. The basic scheme of the proof of the theorem follows that given in [6] and is a formalization of a heuristic notion of A. Markoff. The derivation of the result will be given in detail and in several stages, even though this will duplicate much of the discussion of [6].

Theorem. Under assumptions $(A),(B)$, and $(C)$

$$
W^{-1 / 2} \int_{0}^{T} w_{T}(t) x(t) d t
$$

is asymptotically normally distributed with mean zer" and variance

$$
2 \pi \int_{-\infty}^{\infty} f(\lambda) d M(\lambda) .
$$

Let

$$
S(T)=\int_{0}^{T} w_{T}(t) x(t) d t
$$

and set

$$
\begin{aligned}
U_{i}(T) & =\int_{i[p(T)+q(T)\}}^{(i+1) p(T)+i a(T)} w_{T}(t) x(t) d t, \\
V_{i}(T) & =\int_{(i+1) p(T)+i a(T)}^{(i+1)[p(T)+q(T)]} w_{T}(t) x(t) d t,
\end{aligned}
$$

$j=0,1, \cdots, k-1$. Here $k[p(T)+q(T)]=T$ and the numbers $k=k(T), p(T), q(T)$ will be chosen so that $k, p, q \rightarrow \infty$ and $q(T) / p(T) \rightarrow 0$ as $T \rightarrow \infty$. In effect, the interval $[0, T]$ is being divided into an alternating succession of big blocks (length $p(T)$ ) and small blocks (length $q(T)$ ). The $U_{i}$ 's are the large block integrals and the $V_{i}$ 's the small block integrals. We first show that the contribution of the small block integrals is negligible. Now

$$
\begin{aligned}
E\left|\sum_{i=1}^{k} V_{i} W^{-1 / 2}\right|^{2}=E\left|\int_{A(T)} w_{T}(t) x(t) d t W^{-1 / 2}\right|^{2} \\
\leq \int|r(u)| \int_{A(T)}\left|w_{T}(t+u) w_{T}(t)\right| d t d u W(T)^{-1} \\
\leq \int|r(u)|\left[\int_{A(T)}\left|w_{T}(t+u)\right|^{2} d t \int_{A(T)}\left|w_{T}(t)\right|^{2} d t\right]^{1 / 2} W(T)^{-1},
\end{aligned}
$$


where

$$
A(T)=\bigcup_{i=1}^{k}\{t \mid j p(T)+(j-1) q(T) \leq t \leq j(p(T)+q(T))\} .
$$

But (3.6) must approach zero because of the absolute integrability of $r(u)$ and part $2 a$ of assumption $(A)$. But this means that

$$
\sum_{1}^{k} V_{i} W^{-1 / 2} \rightarrow 0
$$

in probability as $T \rightarrow \infty$.

Our object now is to show that

$$
\sum_{1}^{k} U_{i} W^{-1 / 2}
$$

is asymptotically normally distributed with mean zero and variance (3.2) as $T \rightarrow \infty$. The theorem on asymptotic normality of $S(T) W^{-1 / 2}$ would then follow immediately for (3.7) is asymptotically negligible. Let us introduce for this purpose the distribution functions

$$
G_{i, r}(x)=P\left\{U_{i} W^{-1 / 2} \leq x\right\}
$$

and the events

$$
A\left(j, T, m_{i}, \delta\right)=\left\{m_{i} \delta<U_{i} W^{-1 / 2} \leq\left(m_{i}+1\right) \delta\right\} .
$$

Now

$$
\begin{aligned}
\sum_{\left(m_{1}+\cdots+m_{k}+k\right) 8 \leq x} P\left(\bigcap_{i=1}^{k} A\left(j, T, m_{i}, \delta\right)\right) & \leq P\left(\sum_{i=1}^{k} U_{i} W^{-1 / 2} \leq x\right) \\
& \leq \sum_{\left(m_{1}+\cdots+m_{k}\right) 8 \leq x} P\left(\bigcap_{i=1}^{k} A\left(j, T, m_{i}, \delta\right)\right) .
\end{aligned}
$$

Notice that

$$
P\left(\max _{i=1, \cdots, k}\left|U_{i} W^{-1 / 2}\right|>\tau_{k}\right)<\epsilon,
$$

where $\tau_{k}=(k C / \epsilon)^{1 / 2}$ with $C$ a constant. This follows from the fact that

$E\left|\max _{i=1, \cdots, k}\right| U_{i} W^{-1 / 2}||^{2} \leq E\left|\sum_{1}^{k}\right| U_{i} W^{-1 / 2}||^{2}$

$$
\leq W(T)^{-1}\left(\sum_{i=1}^{k} E^{1 / 2}\left|U_{i}\right|^{2}\right)^{2} \leq k C
$$

and an application of the Tchebycheff inequality.

Further

$$
\begin{array}{r}
\left|\sum_{\left(m_{1}+\cdots+m_{k}\right) \delta \leq x} P\left(\bigcap_{1}^{k} A\left(j, T, m_{i}, \delta\right)\right)-\sum_{\left(m_{1}+\cdots+m_{k}\right) \delta \leq x} \prod_{1}^{k} P\left(A\left(j, T, m_{i}, \delta\right)\right)\right| \\
\leq k\left(\frac{2 \tau_{k}}{\delta}\right)^{k} g(q(T))+2 \epsilon .
\end{array}
$$


For the probability contributed by all the sets $\bigcap_{i=1}^{k} A\left(j, T, m_{i}, \delta\right)$ for which max $\left|U_{i} W^{-1 / 2}\right|>\tau_{k}$ is by (3.12) at most $\epsilon$. Consider now all the sets $\bigcap_{i=1}^{k} A\left(j, T, m_{j}, \delta\right)$ for which $\max \left|U_{i} W^{-1 / 2}\right| \leq \tau_{k}$. By repeated application of the mixing condition $(B)$ it is clear that

$$
\mid P\left(\bigcap_{i=1}^{k} A\left(j, T, m_{i}, \delta\right)\right)-\prod_{i=1}^{k} P\left(A\left(j, T, m_{i}, \delta\right) \mid \leq k g(q(T)) .\right.
$$

Since there are $\left(2 \tau_{k} / \delta\right)^{k}$ sets of this form, the desired inequality (3.14) is obtained.

Inequality (3.14) will later be applied to show that the sum of the $U_{i} W^{-1 / 2}$ has the same asymptotic distribution as the sum of independent random variables with the same marginal distributions, as long as $k(T), q(T), p(T)$ are appropriately chosen. However, let us see what the asymptotic distribution of the sum of such independent random variables would be. The distribution function of these $k$ independent random variables is

$$
G_{1, T} * \cdots * G_{k, T}(x)
$$

the convolution of $G_{1, T}(x), \cdots, G_{k, T}(x)$.

Lemma. The distribution function (3.16) is asymptotically normally distributed with mean zero and variance (3.2).

Now

$$
\begin{aligned}
\sum_{j=1}^{k} E\left|U_{i} W^{-1 / 2}\right|^{2} & \rightarrow \int_{-\infty}^{\infty} r(u) \rho(u) d u \\
& =2 \pi \int_{-\infty}^{\infty} f(\lambda) d M(\lambda)
\end{aligned}
$$

as $T \rightarrow \infty$ by parts $2 a$ and 3 of assumption $(A)$. Further (3.17) is positive since $f(\lambda)$ is positive everywhere. By Liapounov's form of the central limit theorem [4], if

$$
\sum_{i=1}^{k} E\left|U_{i} W^{-1 / 2}\right|^{4}=o\left(\sum_{i=1}^{k}\left|U_{i} W^{-1 / 2}\right|^{2}\right)^{2}
$$

the expression (3.16) is asymptotically normal with mean zero and variance (3.2). But

$$
\begin{gathered}
E\left|U_{i} W^{-1 / 2}\right|^{4}=W\left(T^{T}\right)^{-2} \iiint \int_{i(p(T)+q(T) 1}^{(i+1) p(T)+i a(T)} w_{T}\left(t_{1}\right) w_{T}\left(t_{2}\right) w_{T}\left(t_{3}\right) w_{T}\left(t_{4}\right) \\
{\left[R\left(t_{1}-t_{2}\right) R\left(t_{3}-t_{4}\right)+R\left(t_{1}-t_{3}\right) R\left(t_{2}-t_{4}\right)\right.} \\
\left.+R\left(t_{1}-t_{4}\right) R\left(t_{2}-t_{3}\right)+Q\left(t_{2}-t_{1}, t_{3}-t_{1}, t_{4}-t_{1}\right)\right] d t_{1} d t_{2} d t_{3} d t_{4} .
\end{gathered}
$$

The sum over $j$ of the first three terms on the right hand side of equality (3.19) is

$$
3 \sum_{i=1}^{k} E^{2}\left|U_{i} W^{-1 / 2}\right|^{2} \text {. }
$$

By part $2 a$ of assumption $(A), E\left|U_{i} W^{-1 / 2}\right|^{2}$ approaches zero uniformly in $j$. This coupled with (3.17) implies that expression (3.20) approaches zero as $T \rightarrow \infty$. Consider now the last term on the right hand side of (3.19). By part 2 of assumption $(A)$ and the absolute integrability of $Q$, the sum over $j$ of the last term on the right of (3.19) tends to zero as $T \rightarrow \infty$. Liapounov's condition for the central limit theorem is therefore satisfied and the Lemma is established. 
Notice that

$$
\begin{aligned}
G_{1, T} * \cdots * G_{k, T}(x-k \delta) \leq & \sum \prod_{\substack{i=1 \\
\left(m_{1}+\cdots+m_{k}+k\right) \delta \leq x}}^{k} P\left(A\left(j, T, m_{i}, \delta\right)\right) \\
& \leq \sum \prod_{\substack{i=1 \\
\left(m_{1}+\cdots+m_{k}\right) \delta \leq x}}^{k} P\left(A\left(j, T, m_{i}, \delta\right)\right) \leq G_{1 . T} * \cdots * G_{k, T}(x+k \delta) .
\end{aligned}
$$

The Lemma coupled with (3.7), (3.11), (3.14) and (3.21) implies the desired theorem if $\delta(T), k(T), p(T), q(T)$ can be chosen so that

$$
\begin{aligned}
& k(T)\left[p\left(T^{\prime}\right)+q(T)\right]=T \\
& k(T), p\left(T^{\prime}\right), q(T) \rightarrow \infty \\
& q(T) / p(T) \rightarrow 0 \\
& k(T) \delta(T) \rightarrow 0 \\
& k\left(\frac{2 \tau_{k}}{\delta}\right)^{k} g\left(q\left(T^{\prime}\right)\right) \rightarrow 0 .
\end{aligned}
$$

The condition $k(T) \delta(T) \rightarrow 0$ is easily satisfied if we set $\delta=k^{-2}$. The difficult condition to satisfy is the last one. Now

$$
k\left(2 \tau_{k} / \delta\right)^{k} \leq k^{s k} D^{k}
$$

where $D=2(C / \epsilon)^{1 / 2}$. Given the existence of a function $g$ satisfying assumption $(B)$, it can always be taken so that

If $k$ is chosen so that

$$
g(u)>(u+1)^{-1}
$$

$$
k \leq[-\log g(q(T))]^{1 / 2}
$$

the last of the conditions (3.22) is satisfied. Keeping these remarks in mind, it is clear that if one takes $q(T)=T^{1 / 2}$ for large $T$, all the conditions (3.22) can be satisfied. The proof of asymptotic normality is complete.

4. Some examples. As noted in the introduction, the general expectation is that the output of a narrow band-pass filter ought to be approximately normally distributed. The theorem obtained in Sec. 3 indicates a fairly broad domain within which this expectation is realized. Our object here is to present a few examples that would roughly describe boundaries outside of which approximate normality would not be valid .

It is clear that the filtered process generally ought to be ergodic at least if the output of the filter is to be approximately normal. We present a simple example to show that ergodicity alone is not enough. Let $f(t)$ be a bounded continuous function with period one and

$$
\int_{0}^{1} f(t) d t=0, \quad \int_{0}^{1} f(t) \cos 2 \pi t d t \neq 0 .
$$

Let the process $x(t)$ be given by

$$
x(t)=f(t+\alpha),
$$


where $\alpha$ is a random variable uniformly distributed on $[0,1]$. The process $x(t)$ is ergodic with mean value

$$
E x(t)=\int_{0}^{1} f(t+\alpha) d \alpha \equiv 0 .
$$

Consider the narrow band-pass filter about $2 \pi$

$S\left(T^{\prime}\right)=\int_{0}^{T} x(t) \cos 2 \pi t d t=[T] \int_{0}^{1} f(t+\alpha) \cos 2 \pi t d t+\int_{[T]}^{T} x(t) \cos 2 \pi t d t$.

Here $[T]$ denotes the largest integer less than or equal to $T$. Now

$$
\lim _{T \rightarrow \infty} \frac{1}{T} \int_{0}^{T} x(t) \cos 2 \pi t d t=\int_{0}^{1} f(t+\alpha) \cos 2 \pi t d t
$$

which is non-normal. However, it should be noted that this process $x(t)$ is not mixing since it has a jump in its spectrum at $2 \pi$.

The second example indicates that if all assumptions of the theorem except for the positivity of the spectral density at the frequency at which the filter is set up are satisfied, the conclusion of the Theorem may not hold. Let $\xi(t)$ be a homogeneous differential process with non-normal increments. Take

$$
x(t)=\xi(t)-2 \xi(t-1)+\xi(t-2) .
$$

Consider the narrow band-pass filter

$$
\int_{0}^{T} x(t) d t
$$

about zero. Now

$$
\int_{0}^{T} x(t) d t=\int_{T-1}^{T}[\xi(t)-\xi(t-1)] d t-\int_{-1}^{0}[\xi(t)-\xi(t-1)] d t
$$

so that the output is not normal. Assumptions $(A),(B),(C)$ are satisfied except in that $f(\lambda)$ has a high order contact with zero at $\lambda=0$.

5. Acknowledgment. The problem discussed in these comments was suggested by S. P. Lloyd. Much of what is relevant in the comments is based on stimulating discussions with S. P. Lloyd and D. Slepian.

\section{REFERENCES}

1. J. L. Doob, Stochastic processes, John Wiley \& Sons, New York, 1953

2. U. Grenander and M. Rosenblatt, Statistical analysis of stationary time series, John Wiley \& Sons, New York, 1957

3. E. Hopf, Ergodentheorie, Chelsea, 1948

4. M. Lodve, Probability theory, Van Nostrand, New York, 1955

5. T. A. Magness, Spectral response of a quadratic device to non-Gaussian noise, J. Appl. Phys. 25, 13571365 (1954)

6. M. Rosenblatt, $A$ central limit theorem and a strong mixing condition, Proc. Natl. Acad. Sci. U. S. A. 42, 43-47 (1956) 